\title{
Percepción de los agentes educativos respecto de la atención a la diversidad en establecimientos educativos
}

\author{
Perception of Educational Agents in Relation to Attention to Diversity \\ in Educational Establishments
}

\section{Percepções dos educadores sobre a atenção à diversidade nos estabelecimentos de ensino}

\begin{abstract}
Carolina Genoveva de las Mercedes Cornejo-Valderrama ${ }^{1}$
Universidad Católica del Maule

Talca, Chile

ccornejo@ucm.cl

Eduardo Segundo Olivera-Rivera ${ }^{2}$

Asesor Técnico Educacional

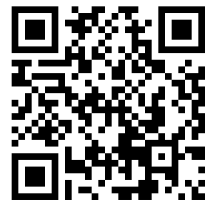

Talca, Chile

esorolivera@gmail.com

Nancy Fabiola Lepe-Martínez $z^{3}$

Universidad Católica del Maule

Talca, Chile

nlepe@ucm.cl

http://orcid.org/0000-0003-3574-044X

Rubén Vidal-Espinoza

Universidad Cardenal Silva Henríquez

Talca-Chile

rvidale@gmail.com

\footnotetext{
${ }^{1}$ Doctora en Educación de la Universidad de Playa Ancha (Chile). Magister en Educación Especial (PUCCh-Chile) Postítulo Educación Especial (UCM-Chile) y Profesora de Educación General Básica (PUCCh-Chile). Académica e investigadora del Departamento de Diversidad e Inclusividad Educativa de la Facultad de Ciencias de la Educación de la Universidad Católica del Maule. Línea de desarrollo académico: Discapacidad intelectual. Línea de investigación: Inclusión, diversidad e interculturalidad, aprendizaje y evaluación.

${ }^{2}$ Doctor en Educación con énfasis en Currículum y Evaluación por la Universidad de Valladolid (España), Magíster en Administración Educacional con mención en Gestión de Sistemas Educativos por la Universidad de Playa Ancha (Chile), Consejero Educacional y Vocacional por la Pontificia Universidad Católica de Chile, Licenciado en Educación con mención en Orientación Educacional por la Universidad Católica del Maule (Chile). Actualmente asesor técnico educacional habilitado por el Ministerio de Educación Pública de Chile. Ha ejercido la docencia investigativa universitaria por 21 años en formación inicial de profesorado para Chile. Posee publicaciones en revistas indexadas nacionales e internacionales. Miembro de la Red de

${ }^{3}$ Doctora (c) en Psicología, Universidad de Concepción; Magíster en Educación, Mención Gestión del Currículum y pos título en Computación Educacional de la Universidad del Bio Bío. Profesora de Educación Especial y Diferenciada de la Universidad Católica del Maule. Académica e investigadora del Departamento de Diversidad e Inclusividad Educativa de la Facultad de Ciencias de la Educación de la Universidad Católica del Maule. Líneas de investigación: Funciones cognitivas básicas en niños, inclusión educativa, estrategias de enseñanza y aprendizaje, identidad docente, pensamiento crítico de estudiantes de universidad y en la formación de docentes. Autora y coautora de variados artículos.

${ }^{4}$ Magister en Educación Especial y Diferenciada de la Pontificia Universidad Católica de Chile. Académico e investigador del Departamento de Diversidad e Inclusividad Educativa de la Facultad de Ciencias de la Educación de la Universidad Católica del Maule. Línea de desarrollo académico: Discapacidad intelectual. Línea de investigación la inserción laboral de jóvenes con discapacidad intelectual con la estrategia del empleo con apoyo (ECA).
} Orientadores Educacionales Latinoamericana y la Sociedad Hispana de Investigadores Científicos con sede en Perú.
\end{abstract}


doi: http://dx.doi.org/10.15359/ree.21-3.17

URL: http://www.una.ac.cr/educare

CORREO: educare@una.cr

Recibido 23 de marzo de 2016 • Corregido 7 de agosto de 2017 • Aceptado 26 de agosto de 2017 Received 23 de marzo de 2016 • Revised 7 de agosto de 2017 • Accepted 26 de agosto de 2017 Recebido 23 de marzo de 2016 • Revisado 7 de agosto de 2017 • Aprovado 26 de agosto de 2017

Resumen: Este artículo tiene como objetivo conocer la percepción de los agentes educativos respecto de la atención a la diversidad de establecimientos educacionales de las comunas de Talca y Curicó (Chile). La muestra está compuesta por 446 profesionales de la educación, agrupados en personal directivo, docente de aula y de apoyo a niños, niñas y jóvenes que presentan necesidades educativas especiales. El método utilizado es no experimental, con propósitos descriptivos, transaccional, se usa un cuestionario como instrumento de recogida de información. El análisis de la información se realiza a través de tablas de frecuencia. Como conclusión se puede indicar que los agentes educativos tienen una percepción favorable en cuanto a la atención a la diversidad, el concepto de diversidad lo ven como algo enriquecedor que permite la flexibilización del currículo, el trabajo interdisciplinario, ajustar las exigencias curriculares a las necesidades estudiantiles para generar oportunidades de aprendizaje a todo el estudiantado.

Palabras claves: Atención a la diversidad; inclusión educativa; agentes educativos; percepción de agentes educativos.

Abstract: This article aims to know the perception of educational agents in relation to the attention to diversity in educational establishments in the villages of Talca and Curicó (Chile). The sample was made up of 446 education professionals, grouped into managers, classroom teachers, and professionals in counseling for children and youth with special educational needs. The method implemented was non-experimental, for descriptive purposes, transactional, and a questionnaire was used as instrument for collecting information. The analysis of the information was done through frequency tables. As a conclusion, it can be said that educational agents have a favorable perception in relation to the attention to diversity; the concept of diversity is seen as an enriching element that allows flexibility of the curriculum, the interdisciplinary work, adjustment of the curricular requirements to the needs of students to generate learning opportunities for all students.

Keywords: Attention to diversity; educational inclusion; educational agents; perception educational agents.

Resumo: O objetivo desse artigo é conhecer a percepção dos educadores no que se refere à atenção a diversidade, nos estabelecimentos de ensino dos municípios de Talca e Curicó (Chile). Fizeram parte da amostra 446 profissionais da educação, divididos em diretores, professores e comitê de apoio as crianças e jovens que tinham alguma necessidade educativa especial. O método utilizado é não experimental, descritivo, transacional. Utilizou-se também um questionário como instrumento para a recolecção de informações. A análise das informações é feita através de tabelas de frequência. Em conclusão, o que indica a pesquisa é que os educadores têm uma percepção positiva sobre a atenção à diversidade; o conceito de diversidade é visto como enriquecedor, algo que permite a flexibilidade no currículo, o trabalho interdisciplinar, o ajuste das exigências curriculares às necessidades dos alunos com o fim de gerar oportunidades de aprendizagem para todos os estudantes.

Palavras-chave: atenção à diversidade; inclusão educacional; educadores; percepção dos agentes educacionais. 


\section{Introducción}

La variedad de conceptos sobre "educación" y una búsqueda constante de mejoras del sistema educativo han conducido a reformas en la mayoría de los países, y a la incorporación de los conceptos de calidad y equidad educativa. En Chile, están considerados como principios de la Ley Núm. 20.370 (MINEDUC, 2009b).

La educación considera ejes claves para el desarrollo integral el desarrollo cognitivo y valórico del estudiantado. Barreiro (1969) señala que "la educación verdadera es praxis, reflexión y acción del hombre sobre el mundo para transformarlo" (p. 7). Lo anterior se explica a partir de las consideraciones que se realizan respecto de la educación, al pensarla como una experiencia social, a través de la cual el ser humano se conoce y enriquece a sí mismo al interactuar con su entorno, generando aprendizajes junto a otros individuos.

En Chile, la educación es un derecho de todas y todos, y es independiente de las condiciones y características de las personas; está consignada en la Constitución Política de la República de Chile de 1980 (Gobierno de Chile, 1998) y en las leyes y normativas que de ellas se derivan y, de manera particular, en las del ámbito educativo.

Desde un análisis amplio del concepto de educación, emana la diversidad, implicando cambios culturales, la transformación del pensamiento y de la realización de las prácticas pedagógicas que permitirán considerar las diferencias individuales al interior de la sala de clase.

La atención educativa a la diversidad se organiza desde políticas y normativas con características más integracionistas que, desde un enfoque inclusivo (Ministerio de Educación, 2007, 2009a; Ministerio de Planificación, 2010), otorga recursos y apoyos económicos y técnicos para la implementación del Programa de Integración Escolar (PIE) que se encuentra instalado en las escuelas hace más de dos décadas. Significa nuevos desafíos para los distintos estamentos educacionales y reorientaciones en el rol y en las modalidades tradicionales de funcionamiento de las instituciones escolares.

La escuela es reconocida como el espacio central de una comunidad, un lugar de encuentro con la cultura y la educación formal; además, de ser el lugar que genera modelos positivos de interacciones, de relaciones respetuosas y productivas, entre las distintas personas que a ella asisten, sin distinciones de ningún tipo.

Por tanto, es imprescindible garantizar una escuela de calidad, es decir, una escuela que reconoce y respeta los derechos de los niños, niñas y jóvenes, que se convierte en una comunidad educativa que se compromete a realizar, de modo continuo y permanente, procesos de reflexión en torno a las concepciones y percepciones que cada integrante de la comunidad educativa tiene en relación con la atención a la diversidad en contextos educativos y, de este modo, generar conceptualizaciones compartidas que luego se vean reflejadas en prácticas educativas 
doi: http://dx.doi.org/10.15359/ree.21-3.17

URL: http://www.una.ac.cr/educare

CORREO: educare@una.cr

de calidad, posibilitando así el acceso, permanencia y egreso exitoso del estudiantado de los sistemas educativos

En este sentido, indagar y develar las percepciones que tienen los distintos actores del sistema educativo permitirá generar conocimiento útil para orientar tanto la política pública relacionada con la atención a la diversidad en contextos educativos, como la gestión directiva y la didáctica de aula que posibiliten procesos educativos de mejor calidad y más inclusivos.

\section{Atención a la diversidad}

El Ministerio de Educación de Chile (MINEDUC), desde la década de los años noventa, a través de los procesos de reforma educacional, ha elaborado e implementado diversas acciones para favorecer los accesos y la calidad de los aprendizajes de todos los niños y niñas del sistema escolar. Ha favorecido, de manera particular, a los colectivos más diversos, y entre ellos los que presentan necesidades educativas especiales (NEE), en contextos de escuelas regulares y aulas comunes, otorgando espacios educativos de participación y de experiencias de aprendizaje.

En este marco, la comunidad educativa tiene un desafío permanente en el diseño de respuestas educativas que permitan abordar las necesidades de aprendizaje de cada estudiante, para la generación de aprendizajes significativos, lo que demanda nuevas formas de hacer gestión directiva y de aula que permitan, efectivamente, atender la diversidad estudiantil. Lo anterior debiera promover cambios de orden organizativo, metodológico y curricular; asimismo, demanda recursos profesionales, materiales y de formación. Se convierte en una tarea permanente para toda la comunidad educativa y también en un obstáculo cuando no se cuenta con una organización y optimización de los recursos técnico-pedagógicos de manera participativa y colaborativa.

Para abordar eficientemente esta realidad, se hace imprescindible el trabajo multidisciplinario en los establecimientos, la participación de la familia y de la comunidad, a través del proceso educativo, que permita la interacción y articulación de las capacidades de cada una de las personas en favor de los aprendizajes de todo el estudiantado y particularmente de aquel que presenta necesidades educativas especiales, y brindar una respuesta ajustada y diversificada a la realidad educativa. De este modo, es posible avanzar en el cumplimiento de los principios orientadores de la Ley General de Educación (MINEDUC, 2009b), a través de propuestas basadas en el respeto a la diversidad y en enfoques más heterogéneos, "considerando la autonomía de los establecimientos educacionales, promoviendo y valorando las diferencias culturales" (Ministerio de Educación, 2015, p. 9).

Chile ha generado diversas normativas en favor de la niñez y la juventud que presentan algún tipo de NEE durante su etapa escolar; entregando asimismo diversos tipos de apoyos 
y recursos, beneficiando no solo al alumnado, sino también aal personal docente de aula. Todo esto, con el propósito de ir reconociendo en la práctica educativa la individualidad del estudiantado y evitar tendencias homogeneizadoras y poco flexibles.

Otro elemento importante de considerar en el entorno educativo son los efectos o repercusiones que se observan producto de la ampliación de la población que tiene acceso a la educación; la obligatoriedad de asistir a la escuela hasta los 18 años y la incorporación en las aulas regulares de niños, niñas y jóvenes que presentan diferencias culturales, sociales, cognitivas y físicas. Esto constituye un desafío complejo para los distintos actores de la escuela regular, situaciones que requieren de cambios y modificaciones en la gestión organizacional y en el desempeño profesional, como asimismo en los significados y representaciones culturales que se tienen por los conceptos de "normalidad" y "diversidad" al interior de cada una de las instituciones educativas y de cada integrante de estas.

En este sentido, el concepto de diversidad, etimológicamente, significa variedad, desemejanza o gran cantidad de varias cosas distintas (RAE, 2014), mientras que la diferencia alude a la variedad entre cosas de una misma especie. Como especie humana, se comparte una variedad de características comunes que nos asemejan y, al mismo tiempo, cualidades distintas, que nos hacen seres únicos e irrepetibles. Sin embargo, existe una tendencia a considerar la diferencia como aquello que se distancia o desvía de la "mayoría", de lo "normal" o "frecuente", es decir, desde criterios normativos. Las diferencias son una condición de la naturaleza humana, pero su valoración, positiva o negativa, es algo externo a la persona.

La respuesta educativa en la atención a la diversidad se puede comprender como una variedad de actividades pedagógicas conducentes al aseguramiento de la equidad educativa, a través de un trabajo planificado, implementado, ejecutado y evaluado sistemáticamente dirigido al alumnado que presenta diferencias evidenciadas en los resultados de aprendizajes escolares.

Por ello, la escuela debe considerar la diversidad como una oportunidad de crecimiento profesional, que permite el diseño y la implementación de diversas acciones académicas que brindan espacios de respeto individual a las características particulares de cada estudiante. De este modo, se garantiza la igualdad de oportunidades con la realización de una gestión técnicapedagógica adecuada y pertinente a la realidad escolar. Se demuestra así, la conformación de una comunidad de aprendizaje, una escuela abierta, que se van construyendo entre todos sus miembros, que la conforman -personal docente, alumnado, padres y madres e instituciones de la comunidad- que van permitiendo un funcionamiento y participación colaborativa y responsable desde un accionar positivo y solidario.

Por tanto, el constructo de diversidad involucra a la escuela en la realización de numerosos trabajos de carácter preventivo y de intervención, como respuestas al alumnado que presenta necesidades educativas especiales, es decir, cuando el alumnado "precisa ayudas y recursos adicionales, ya sean humanos, materiales o pedagógicos, para conducir su proceso de desarrollo 
doi: http://dx.doi.org/10.15359/ree.21-3.17

URL: http://www.una.ac.cr/educare

CORREO: educare@una.cr

y aprendizaje, y contribuir al logro de los fines de la educación" (MINEDUC, 2009b, p. 8); y requiere de actuaciones específicas en los ámbitos de aprendizaje escolar, social y de inclusión en el entorno en el que se encuentran.

Crear un entorno de aprendizaje inclusivo ayudará a todos los niños a aprender y realizar su potencial. Los sistemas educativos tienen que adoptar planteamientos más centrados en el alumno, con cambios en los planes de estudio, métodos y materiales de enseñanza y sistemas de evaluación y examen. (Organización Mundial de la Salud, OMS, 2011, p. 17)

Del planteamiento anterior, se puede inferir que la educación puede ser un agente de cohesión y de igualdad, si respeta la diversidad de las personas y de los grupos humanos, valorando a cada persona por lo que es y facilitando a cada quien lo que necesita para desarrollar al máximo sus capacidades y construir su propia identidad. La desigualdad se origina cuando se valoran negativamente las diferencias y cuando se establecen jerarquías entre las personas en función de distintos criterios como, por ejemplo, el origen social o cultural, o el nivel de competencias.

Desde la perspectiva ética, la educación debe asegurar la igualdad, pero no la uniformidad, evitando reproducir las desigualdades presentes en nuestra sociedad. En este sentido, la inclusión de cualquier grupo minoritario pasa por una igualdad de derechos y por el respeto de sus libertades, tal como se señala en el Informe a la UNESCO (Delors, 1997):

La educación tiene una doble misión: enseñar la diversidad de la especie humana y contribuir a una toma de conciencia de las semejanzas y la interdependencia entre todos los seres humanos (p. 51). La enseñanza del pluralismo no sólo es una protección contra las violencias, sino también un principio activo de enriquecimiento cultural y cívico de las sociedades contemporáneas. ... La finalidad de la educación debe consistir en lograr que las distintas minorías puedan asumir su propio destino. (p. 28)

Lo anterior implica participar en ambientes democráticos y solidarios; educar en la diversidad es un medio esencial para desarrollar la comprensión mutua, el respeto y la tolerancia, que son los fundamentos del pluralismo, la convivencia y la democracia. De este el sistema educativo debe contener y respetar el principio de unidad/diversidad, tal como lo explicita Morin (2000) “la educación del futuro deberá velar por que la idea de unidad de la especie humana no borre la de su diversidad, y que la de su diversidad no borre la de la unidad" (p. 27).

\section{Metodología}

Este estudio se adscribe al paradigma cuantitativo, centrándose en la descripción de las unidades de análisis dadas por la variable de contexto en la atención a la diversidad que realizan 
los agentes educativos que se desempeñan en establecimientos educacionales de dependencia municipal de las comunas de Talca y Curicó. Es una investigación no experimental, ex post facto, de carácter microsociológica por la amplitud del trabajo de campo.

La muestra se define por la facilidad de los accesos y la voluntariedad de participar en el estudio, por tanto, es intencionada. Está constituida por un total de 446 agentes educativos, de los cuales, el personal directivo conforma el 13,9\%, docente de aula un 65,5\% y profesionales de apoyo a los niños, niñas y jóvenes que presentan necesidades educativas especiales, el 20,6\%. Pertenecen a 30 establecimientos educacionales de los niveles de educación básica y media de las comunas de Curicó y Talca.

En relación con el género, se tiene en la Tabla 1, que en personal directivo hay mayor presencia en el género masculino, y de manera contraria entre docentes de aula y profesionales de apoyo. De manera global, hay un porcentaje mayor dado para el género femenino, alcanzó un $65,2 \%$ del total de la muestra.

Tabla 1: Distribución de la muestra por género y tipo de agente educativo

\begin{tabular}{|c|c|c|c|c|}
\hline \multirow{3}{*}{ Tipo de Agente } & \multicolumn{4}{|c|}{ Genero } \\
\hline & \multicolumn{2}{|c|}{ Hombre } & \multicolumn{2}{|c|}{ Mujer } \\
\hline & $f$ & $\%$ & & $\%$ \\
\hline Personal directivo & 35 & 7,8 & 27 & 6,0 \\
\hline Docentes de aula & 110 & 24,7 & 182 & 40,8 \\
\hline Profesionales de apoyo & 10 & 2,3 & 82 & 18,4 \\
\hline Total & 155 & 34,8 & 291 & 65,2 \\
\hline
\end{tabular}

Nota: Elaboración propia.

Edad promedio de 46 años, moviéndose los rangos entre 23 y 69 años y una desviación estándar de 11,9. Presenta una media de 21,1 años de experiencia laboral con una desviación estándar de 10,6 y con un rango mínimo de 1 y máximo de 45 años. Llevan en promedio 9,3 años trabajando en la unidad educativa y una desviación estándar de 7,9.

En cuanto a la formación profesional (Tabla 2), se puede señalar que el 13,9\% es personal directivo y ocupa cargos de dirección, subdirección e inspección general; con una variedad de formación inicial, pero que de manera mayoritaria corresponde al título de profesorado. 
doi: http://dx.doi.org/10.15359/ree.21-3.17

URL: http://www.una.ac.cr/educare

CORREO: educare@una.cr

Tabla 2: Formación profesional de participantes a nivel de título inicial

\begin{tabular}{|c|c|c|c|}
\hline Agentes educativos & & $f$ & $\%$ \\
\hline \multirow[t]{7}{*}{ Personal directivo } & Profesorado de Educación Básica & 32 & 7,2 \\
\hline & Profesorado de Educación Media o de Estado & 21 & 4,7 \\
\hline & Profesorado de Educación Especial & 6 & 1,4 \\
\hline & Educación de Párvulo & 1 & 0,2 \\
\hline & Orientación Vocacional & 1 & 0,2 \\
\hline & Técnico Administrativo & 1 & 0,2 \\
\hline & Sub-Total & 62 & 13,9 \\
\hline \multirow[t]{4}{*}{ Docentes de aula } & Profesorado de Educación Básica & 34 & 7,7 \\
\hline & Profesorado de Educación Media o de Estado & 176 & 39,4 \\
\hline & Educación de Párvulo & 82 & 18,4 \\
\hline & Sub-Total & 292 & 65,5 \\
\hline \multirow[t]{8}{*}{ Profesionales de apoyo } & Profesorado de Educación Especial & 45 & 10,1 \\
\hline & Psicopedagogía & 13 & 2,9 \\
\hline & Psicólogía & 12 & 2,7 \\
\hline & Fonoaudiólogía & 9 & 2,0 \\
\hline & Kinesiólogía & 5 & 1,1 \\
\hline & Orientación Familiar & 2 & 0,4 \\
\hline & Trabajo Social & 6 & 1,4 \\
\hline & Sub-Total & 92 & 20,6 \\
\hline TOTAL & & 446 & 100 \\
\hline
\end{tabular}

Nota: Elaboración propia.

Un segundo grupo de participantes se clasifica en docentes de aula, conformado por 292 personas, correspondiendo al 65,5\% del total de la muestra. Es profesorado de educación media o de Estado, de educación básica y de párvulo.

El último grupo conformado por profesionales de apoyo a la educación corresponde al $20,6 \%$, integrado por una variedad de personal experto en áreas específicas que cooperan en el fortalecimiento y aseguramiento de los aprendizajes de los niños, niñas y jóvenes que asisten a los diversos establecimientos educacionales y que participan en el Programa de Integración Escolar. 
Del personal docente directivo, el $80,6 \%$ ha realizado programas de perfeccionamiento en la línea de la formación continua (Tabla 3) y el 30,6\% está en posesión del grado de magíster.

Tabla 3: Formación continua y posgrado de participantes

\begin{tabular}{lcccccc}
\hline & \multicolumn{2}{c}{ Personal directivo (62) } & \multicolumn{2}{c}{ Docentes de aula (292) } & \multicolumn{2}{c}{ Profesionales de apoyo (92) } \\
\hline & $\mathrm{f}$ & $\%$ & $\mathrm{~F}$ & $\%$ & $\mathrm{f}$ & $\%$ \\
Pos-título & 50 & 80,6 & 120 & 41,0 & 54 & 58,7 \\
Licenciatura & 17 & 27,4 & 118 & 40,4 & 65 & 70,7 \\
Magíster & 19 & 30,6 & 32 & 10,9 & - & - \\
\hline
\end{tabular}

Nota: Elaboración propia

Como promedio $(90,0 \%)$ de perfeccionamiento efectuado por el personal docente de aula, conforma de la muestra total el $60,5 \%$ con programas terminados; y solo el 10,9\% cuenta con el grado de magister. Por tanto, el grupo de profesionales de la educación cuenta con título de posgrado conforman el 11,4\% entre los grupos de personal directivo y docentes de aula.

Entreel grupo de profesionales deapoyo solo han realizado formación continua, comprendida como pos-título conformando el 58,7\% y no cuentan con formación de posgrado, en el área específica de magíster o maestrías y doctorados. Se releva que 27 no cuentan con Licenciatura, sin embargo el 85,9\% ha realizado formación específica sobre atención a la diversidad.

Tabla 4: Frecuencia de formación específica en la atención a la diversidad. Muestra Total

\begin{tabular}{lcc}
\hline & $f$ & $\%$ \\
\hline Personal directivo & 10 & 2,2 \\
Docentes de aula & 113 & 25,3 \\
Profesionales de apoyo & 79 & 17,7 \\
\hline TOTAL & 202 & 45,2 \\
\hline
\end{tabular}

Nota: Elaboración propia.

Respecto de la preparación y manejo de las temáticas relacionadas con la atención a la diversidad, que presentan los distintos agentes educativos, se tiene como resultado global un 45,2\% con formación en áreas específicas, tales como trastornos específicos del lenguaje, dificultades del aprendizaje y déficit atencional (Tabla 4), lo que demuestra desinterés por este tipo de perfeccionamientos. 
doi: http://dx.doi.org/10.15359/ree.21-3.17

URL: http://www.una.ac.cr/educare

CORREO: educare@una.cr

Los resultados de formación en lasáreas relacionadas con las necesidades educativas especiales permanentes se pueden visualizar en la Tabla 5, donde se distingue una mayor preparación en el área de la discapacidad intelectual con un $31,6 \%$ y con porcentajes similares en el conocimiento de personas que presentan discapacidad motora y trastornos de relación y comunicación. Globalmente, se aprecia un promedio de $88,4 \%$ de un total de 446 agentes educativos con cierto nivel de formación para responder al alumnado que requieren de apoyos específicos.

Tabla 5: Formación específica recibida en NEE permanentes.

Muestra Total

\begin{tabular}{lcc}
\hline NEE Permanente & $\mathrm{f}$ & $\%$ \\
\hline Discapacidad motora & 96 & 21,5 \\
Discapacidad visual & 44 & 9,9 \\
Discapacidad auditiva & 57 & 12,8 \\
Discapacidad relación y comunicación & 104 & 23,3 \\
Discapacidad intelectual & 141 & 31,6 \\
\hline
\end{tabular}

Nota: Elaboración propia.

Asimismo, la Tabla 6 evidencia, que porcentualmente son los grupos profesionales de apoyo (54,3\%) los que han recibido mayor formación en tópicos relacionados con las necesidades educativas permanentes y se destaca la preparación en el área de la discapacidad intelectual, lo que se repite en docentes de aula y personal directivo.

Tabla 6: Formación específica sobre las NEE permanentes por agentes educativos

\begin{tabular}{lcccccc}
\hline & $\begin{array}{c}\text { Personal directivo } \\
(62)\end{array}$ & $\begin{array}{c}\text { Docentes de aula } \\
(292)\end{array}$ & \multicolumn{2}{c}{$\begin{array}{c}\text { Profesionales de apoyo } \\
\text { (92) }\end{array}$} \\
\hline & $\mathrm{f}$ & $\%$ & $\mathrm{f}$ & $\%$ & $\mathrm{f}$ & $\%$ \\
Discapacidad motora & 6 & 9,6 & 61 & 20,8 & 29 & 31,5 \\
Discapacidad visual & 4 & 6,4 & 28 & 9,5 & 12 & 13,0 \\
Discapacidad auditiva & 4 & 6,4 & 31 & 10,6 & 22 & 23,9 \\
Discapacidad intelectual & 10 & 16,1 & 81 & 27,7 & 50 & 54,3 \\
Discapacidad relación y comunicación & 7 & 11,2 & 62 & 21,2 & 35 & 38,0 \\
\hline
\end{tabular}

Nota: Elaboración propia.

10 Carolina Genoveva de las Mercedes Cornejo-Valderrama, Eduardo Segundo Olivera-Rivera, Nancy Fabiola Lepe-Martínez y Rubén Vidal-Espinoza 
En la actualidad, se hace relevante que todo el profesorado reciba formación específica conceptual y práctica, referida a las necesidades educativas especiales y particularmente las de carácter permanente, con base en el conocimiento del término discapacidad, tal como lo explicita la Fundación Nacional de los Discapacitados (s. f.):

Un concepto genérico, amplio, que se relaciona con una determinada condición física, sensorial, mental, intelectual, psiquiátrica o multidéficit, que limita actividades y restringe la participación, determinando por lo general, una relación negativa entre quienes presentamos una o más deficiencias y los factores contextuales que nos rodean. (párr. 5)

De este planteamiento se evidencia la necesidad de producir cambios a nivel del sistema educativo, para eliminar barreras y proporcionar servicios de apoyo asegurando una educación de calidad en entornos inclusivos y participar con sus pares en igualdad deoportunidades educacionales.

La preparación que explicitan el personal docente directivo, docente de aula y profesionales de apoyo en tópicos específicos relacionados con las NEE de carácter transitorio (Tabla 7); evidencia, de manera global, que un $27,1 \%$ ha recibido formación en diversas áreas, lo que favorece el apoyo que brindan a nivel de aula regular. El área de déficit atencional muestra mayor preparación por parte de los sujetos participantes del estudio.

Tabla 7: Formación específica sobre las NEE transitorias. Muestra Total

\begin{tabular}{lcc}
\hline NEE Transitoria & $f$ & $\%$ \\
\hline Trastornos específicos del lenguaje & 106 & 23,7 \\
Coeficiente intelectual límite & 123 & 27,5 \\
Déficit atencional & 141 & 31,6 \\
Dificultades específicas del aprendizaje en lectura & 122 & 27,3 \\
Dificultades específicas del aprendizaje en escritura & 122 & 27,3 \\
Dificultades específicas del aprendizaje en matemáticas & 113 & 25,3 \\
\hline
\end{tabular}

Nota: Elaboración propia.

La Tabla 8 da cuenta de la formación específica que han recibido los distintos agentes educativos, visualizándose un porcentaje mayor en los profesionales de apoyo, sobrepasando el $50 \%$ en cada una de las áreas; seguidos por el profesorado y personal directivo, sin sobrepasar porcentualmente el $26,0 \%$. 
doi: http://dx.doi.org/10.15359/ree.21-3.17

URL: http://www.una.ac.cr/educare

CORREO: educare@una.cr

Estos resultados muestran la falta de preparación de los docentes y las docentes de aula para atender a la diversidad de estudiantes que se encuentran en las aulas regulares, como así también, la carencia comprensiva de los procesos de enseñanza y aprendizaje que permitan producir logros escolares en todo el alumnado.

Tabla 8: Formación específica sobre las NEE transitorias, por agentes educativos

\begin{tabular}{lcccccc}
\hline NEE Transitorias & $\begin{array}{c}\text { Personal directivo } \\
(62)\end{array}$ & $\begin{array}{c}\text { Docentes de aula } \\
(292)\end{array}$ & $\begin{array}{c}\text { Profesionales de apoyo } \\
\text { (92) }\end{array}$ \\
\hline & $f$ & $\%$ & $f$ & $\%$ & $f$ & $\%$ \\
Trastornos específicos del lenguaje & 7 & 11,2 & 48 & 16,4 & 51 & 55,4 \\
Coeficiente intelectual límite & 8 & 12,9 & 66 & 22,6 & 49 & 53,2 \\
Déficit atencional & 13 & 20,9 & 76 & 26,0 & 52 & 56,5 \\
Dificultades Específicas del aprendizaje en lectura & 11 & 17,7 & 61 & 20,8 & 50 & 54,3 \\
Dificultades específicas del aprendizaje en escritura & 11 & 17,7 & 62 & 21,2 & 49 & 53,2 \\
Dificultades específicas del aprendizaje en & 10 & 16,1 & 55 & 18,8 & 48 & 52,1 \\
matemáticas & & & & & &
\end{tabular}

Nota: Elaboración propia.

Los establecimientos educacionales entregan una diversidad de modalidades educativas de atención a la diversidad, en la que participan de manera más directa los grupos profesionales de apoyo y docentes de aula. Ellas son: apoyo al aula común, apoyo en aula de recursos, atención domiciliaria, aula hospitalaria.

Los grupos profesionales de apoyo señalan realizar apoyo específico $(51,1 \%)$ y especializados en el aula de recursos, el 4,3\% solo apoya en el aula común, el 23,9\% realiza apoyo en aula común y aula de recursos y el $20,7 \%$ comparte apoyo específico en aula de recursos y en aula hospitalaria, efectúan también apoyos en domicilio. Estas modalidades son las que aseguran el avance en los aprendizajes escolares respetando las necesidades particulares y asegurando la inclusión educativa.

Las unidades educativas que cuentan con PIE asumen la responsabilidad de asegurar el acceso, la permanencia y el egreso, de cada estudiante que se beneficia directamente. Asimismo, reciben recursos económicos, materiales y de carácter técnico-pedagógico, para apoyar el proceso de aprendizaje de los niños, niñas y jóvenes que presentan necesidades educativas especiales.

Además de los beneficios que aporta el PIE, cuentan con los que otorga la Ley N. 20.248 de Subvención Escolar Preferencial (MINEDUC, 2008). Esta Ley tiene por objetivo mejorar la calidady equidad de la educación en Chile a través de una subvención adicional por estudiante dirigida a 
los establecimientos que atienden la población más vulnerable; además, estos establecimientos se comprometen en el logro de una educación de calidad y mayores grados de equidad.

\section{Instrumento y procedimientos de análisis de datos}

La recolección de datos fue a través de un cuestionario con escala de valoración de 1 al 6 en los conceptos de "nada o nunca", "poco", "algunas veces", "frecuentemente", "bastante" y "siempre"; además de respuesta dicotómica frente a algunos indicadores de existencia. Está basado en Biencinto-López, González-Barbera, García-García, Sánchez-Delgado y Madrid-Vivar (2009), el que fue revisado y se le hicieron modificaciones en algunos indicadores en relación con vocabulario $y$, fue sometido a validación de constructo por el juicio de personas expertas, y al coeficiente de confiabilidad Alfa de Cronbach.

Para el personal docente directivo, el cuestionario obtuvo un índice de confiabilidad por validación de constructo de 0,92; para docentes de aula un 0,90 y para los profesionales de apoyo fue de 0,89. Luego de la aplicación de los cuestionarios, se obtuvo un nivel de fiabilidad calculado con Alfa de Cronbach, resultando un 0,93 en el caso del personal directivo, un 0,91 para docentes de aula y un 0,92 para profesionales de apoyo.

Las dimensiones contempladas en el instrumento son diferenciadas para los distintos agentes educativos, a saber: personal docente directivo (escuela/liceo, intervención en la escuela/liceo, currículo y diversidad), docentes de aula (escuela/liceo, currículo, recursos, nivel de rendimiento y diversidad) y profesionales de apoyo (escuela/liceo, intervención en la escuela/ liceo, currículo, recursos, diagnóstico y diversidad). El análisis de datos fue realizado a través de de estadígrafos descriptivos y de frecuencia con el paquete estadístico SPSS (versión 22).

\section{Resultados}

Se presentan de manera global los resultados agrupados por las dimensiones consideradas en el análisis de la respuesta educativa que expresan los agentes educativos de los distintos establecimientos participantes.

\section{Dimensión escuela/liceo}

Esta dimensión es percibida con conceptos opuestos de "existencia y "no existencia", respecto de temas referidos a la cultura y a la presencia de documentos orientadores propios del establecimiento educacional. Los resultados son bastante similares entre los sujetos participantes de la muestra y se señalan en la Tabla 9. Es el personal docente de aula el que entrega porcentualmente resultados menores en comparación con el directivo y profesionales de apoyo; de todos modos son indicadores positivos, lo que demuestra compromiso con el estudiantado y preocupación por brindar atenciones educativas que le permitan avanzar en sus aprendizajes escolares. 
doi: http://dx.doi.org/10.15359/ree.21-3.17

URL: http://www.una.ac.cr/educare

CORREO: educare@una.cr

Tabla 9: Frecuencia de percepción de los agentes educativos frente a la dimensión Escuela/Liceo

\begin{tabular}{lcccccc}
\hline \multicolumn{1}{c}{ Escuela / Liceo } & $\begin{array}{c}\text { Docentes Directivos } \\
\text { (Existencia) }\end{array}$ & $\begin{array}{c}\text { Docentes de Aula } \\
\text { (Existencia) }\end{array}$ & $\begin{array}{c}\text { Profesionales de Apoyo } \\
\text { (Existencia) }\end{array}$ \\
\hline & $\mathrm{f}$ & $\%$ & $\mathrm{f}$ & $\%$ & $\mathrm{f}$ & $\%$ \\
\hline $\begin{array}{l}\text { [Se trabaja bajo la filosofía de la co- } \\
\text { enseñanza] }\end{array}$ & 39 & 62,9 & 172 & 58,9 & 64 & 69,6 \\
$\begin{array}{l}\text { Existen atenciones educativas } \\
\text { diferenciadas }\end{array}$ & 52 & 83,8 & 203 & 69,5 & 86 & 93,5 \\
$\begin{array}{l}\text { El alumnado promociona de manera } \\
\text { diferente en función de sus capacidades }\end{array}$ & 45 & 72,5 & 206 & 70,5 & 77 & 83,7 \\
$\begin{array}{l}\text { Trabajamos para proporcionar } \\
\text { [oportunidades] a todo el alumnado para } \\
\text { el logro de sus objetivos }\end{array}$ & 57 & 91,9 & 241 & 82,5 & 82 & 89,1 \\
$\begin{array}{l}\text { En ocasiones flexibilizamos los grupos en } \\
\text { función de la actividad }\end{array}$ & 58 & 93,5 & 238 & 81,5 & 86 & 93,4 \\
$\begin{array}{l}\text { Orientamos al alumnado a opciones } \\
\text { profesionales o académicas diferentes } \\
\text { según su capacidad }\end{array}$ & 48 & 77,4 & 232 & 79,4 & 85 & 92,4 \\
$\begin{array}{l}\text { [Toda la escuela/liceo] participa de un } \\
\text { proyecto común y una organización } \\
\text { inclusiva para que todo el alumnado logre } \\
\text { los objetivos }\end{array}$ & 54 & 87,0 & 236 & 80,8 & 75 & 81,5 \\
$\begin{array}{l}\text { Los métodos y las estrategias educativas } \\
\text { están adaptadas a cada estudiante }\end{array}$ & 48 & 77,4 & 217 & 74,3 & 74 & 80,5 \\
\hline
\end{tabular}

Nota: Basado en Biencinto et al. (2009, p. 25, ítem 23, 24, 25, 26, 27, 28, 29 y 30).

\section{Dimensión concepto de diversidad}

Este aspecto es analizado desde la perspectiva de todos los agentes educativos participantes, quienes muestran resultados positivos en relación con el concepto de diversidad, visualizado como algo positivo y enriquecedor y que las diferencias son individuales (91\%); además indican que los tipos de diversidad se crean en función de las necesidades educativas que presenta el estudiantado $(89,2 \%)$.

\section{Dimensión intervención de la escuela/liceo frente a la diversidad}

La percepción que tienen los distintos integrantes que se desempeñan en escuela/liceo de las comunas de Curicó y Talca (Tabla 10), en relación con procesos de intervención educativa

14 Carolina Genoveva de las Mercedes Cornejo-Valderrama, Eduardo Segundo Olivera-Rivera, Nancy Fabiola Lepe-Martínez y Rubén Vidal-Espinoza 
que realizan el personal docente directivo y profesionales de apoyo, es positiva -oscila entre las valoraciones de siempre, mucho y bastante-, cuando enfrentan estudiantes que presentan aprendizajes diversos frente a situaciones educativas.

Frente a los procesos de intervención que se realizan en las unidades educativas, los grupos profesionales de apoyo indican en un $72,9 \%$ y el personal directivo en un $67,7 \%$ que el estudiantado es siempre agrupado de acuerdo con sus características, una vez detectadas sus diferencias.

Tabla 10: Frecuencia de percepción respecto de los procesos de intervención que se realizan en la unidad educativa frente a la diversidad

\begin{tabular}{lcccc}
\hline Intervención de la unidad educativa frente a la diversidad & \multicolumn{2}{c}{$\begin{array}{c}\text { Personal directivo } \\
\text { (siempre) }\end{array}$} & $\begin{array}{c}\text { Profesionales de apoyo } \\
\text { (siempre) }\end{array}$ \\
\hline & $\mathrm{f}$ & $\%$ & $\mathrm{f}$ & $\%$ \\
\hline $\begin{array}{l}\text { Una vez detectadas las diferencias, se agrupa al estudiantado en } \\
\text { función de estas }\end{array}$ & 42 & 67,7 & 67 & 72,9 \\
$\begin{array}{l}\text { Una vez detectadas las diferencias, todo el alumnado permanece } \\
\text { en el mismo grupo y cuentan con los apoyos y adaptaciones nece- } \\
\text { sarias }\end{array}$ & 47 & 75,8 & 81 & 88,0 \\
$\begin{array}{l}\text { Una vez detectadas las diferencias, el alumnado se agrupa de ma- } \\
\text { nera flexible en función del objetivo de la actividad o su nivel, alter- } \\
\text { nan grupos heterogéneos y homogéneos }\end{array}$ & 45 & 72,5 & 79 & 85,9 \\
$\begin{array}{l}\text { [Se integra al alumnado con NEE con el fin de adaptarlo al sistema } \\
\text { educativo] }\end{array}$ & 44 & 70,9 & 90 & 97,8
\end{tabular}

Nota: Basado en Biencinto et al. (2009, p. 26, ítem 35, 36, 37 y 38).

En este sentido, el personal docente directivo declara su disposición en la implementación de actividades curriculares considerando apoyos y recursos adecuados a las características específicas del estudiantado; como también considera (70,9\%) los procesos de integración escolar a quienes presentan necesidades educativas especiales.

\section{Dimensión currículo}

Frente a esta dimensión (Tabla 11) responden todos los agentes educativos participantes (446). Se observa coincidencia entre las percepciones de los integrantes de los diversos establecimientos educativos que demuestran que se están orientando en acciones más inclusivas cuando señalan $(81,1 \%)$ la utilización del currículo nacional para todo el alumnado y la flexibilidad de él permite la realización de las adaptaciones necesarias para que avance en sus aprendizajes. 
doi: http://dx.doi.org/10.15359/ree.21-3.17

URL: http://www.una.ac.cr/educare

CORREO: educare@una.cr

Además, concuerdan en promedio (56\%) en que solo el alumnado que lo requiera de acuerdo con sus necesidades de apoyo y características particulares- recibe atención especializada en el aula de recurso. Lo anterior demuestra una mayor disposición a brindar respuestas educativas especializadas al interior de la sala de clases común $(78,2 \%)$ y una mayor apertura del profesorado para aceptar a otros grupos profesionales y compartir su clase en beneficio de los niños, niñas y jóvenes que presentan necesidades educativas especiales, como también de todo el grupo curso.

Tabla 11: Frecuencia de percepción de los agentes educativos de la dimensión currículo

\begin{tabular}{|c|c|c|c|c|c|c|}
\hline \multirow[t]{2}{*}{ Currículum } & \multicolumn{2}{|c|}{$\begin{array}{l}\text { Personal directivo } \\
\text { (siempre) }\end{array}$} & \multicolumn{2}{|c|}{$\begin{array}{l}\text { Docentes de aula } \\
\text { (siempre) }\end{array}$} & \multicolumn{2}{|c|}{$\begin{array}{l}\text { Profesionales apoyo } \\
\text { (siempre) }\end{array}$} \\
\hline & $f$ & $\%$ & $f$ & $\%$ & $f$ & $\%$ \\
\hline $\begin{array}{l}\text { El currículo es el mismo para la totalidad } \\
\text { incluyendo las adaptaciones necesarias para } \\
\text { obtener unos objetivos mínimos }\end{array}$ & 56 & 90,3 & 229 & 78,4 & 77 & 83,6 \\
\hline $\begin{array}{l}\text { El currículo tiene un núcleo central y común para } \\
\text { la totalidad y permite opciones ... para crear } \\
\text { diferentes itinerarios }\end{array}$ & 51 & 82,2 & 226 & 77,3 & 66 & 71,7 \\
\hline $\begin{array}{l}\text { El alumnado diferente permanece ... con apoyo } \\
\text { especializado [en el] aula [regular junto a sus pares.] }\end{array}$ & 53 & 85,4 & 211 & 72,2 & 85 & 92,3 \\
\hline $\begin{array}{l}\text { El alumnado diferente se encuentra en el aula de } \\
\text { recurso con personal especializado }\end{array}$ & 38 & 61,2 & 156 & 53,4 & 56 & 60,9 \\
\hline $\begin{array}{l}\text { Los currículos se adaptan a las necesidades } \\
\text { puntuales de las situaciones gracias al trabajo } \\
\text { cooperativo de los equipos interdisciplinares }\end{array}$ & 45 & 72,5 & 208 & 71,2 & 76 & 82,6 \\
\hline $\begin{array}{l}\text { La comunidad educativa asume la atención } \\
\text { [del] alumnado [en situación de] discapacidad, } \\
\text { contemplándose en el proyecto educativo y } \\
\text { curricular del [colegio/liceo] }\end{array}$ & 43 & 69,3 & 213 & 72,9 & 86 & 93,5 \\
\hline
\end{tabular}

Nota: Basado en Biencinto, et al. (2009, p. 26, ítem 46, 47, 48, 49, 50 y 51).

Importa destacar la valoración positiva que se otorga al trabajo de equipos interdisciplinarios (73,7\%) y la explicitación de acciones educativas que refuerzan y potencian procesos inclusivos, cuando declaran que se asume la atención a la diversidad y particularmente frente a estudiantes en situación de discapacidad en el proyecto educativo institucional de sus unidades educativas (76,6\%). 


\section{Recursos}

Esta dimensión es analizada (Tabla 12) desde la existencia del recurso, como también la frecuencia de su uso, desde la perspectiva de docentes de aula y profesionales de apoyo, en una muestra de 384 participantes.

En cuanto a la percepción que tienen de sus unidades educativas, se observan algunos datos dicotómicos entre los grupos participantes. En este sentido, el 73,9\% señala que cuenta con apoyo del profesorado especialista en el aula, sin embargo, el 56,5\% explica que la frecuencia de ese apoyo en alguna asignatura específica fluctúa entre los rangos de bastante, mucho y siempre. Difieren de manera significativa los grupos profesionales especialistas, señalan 91 $(98,9 \%)$ la realización de apoyo al interior de la clase y con una frecuencia del 85,8\%.

Asimismo, el 58,5\% indica que en sus colegios existen aulas de recursos o de apoyo pedagógico y solo el 44,8\% señala que se usa siempre, a diferencia de la opinión de los grupos profesionales de apoyo; conjuntamente, declaran un 79,3\% la existencia de aula de apoyo pedagógico para la mejora de las competencias lingüísticas del alumnado, pero la frecuencia de la realización de ese apoyo específico solo alcanza un 18,5\% y es el profesorado el que expresa una opinión levemente más favorable (46,9\%).

Se observa coincidencia en reconocer que los docentes y las docentes de aula agrupan al estudiantado en función de los distintos niveles de rendimiento (64\%) y la frecuencia del cumplimiento de ese indicador es en promedio un 45\%. Asimismo, indican que cuentan con planes de acción tutorial (66\%), como un medio para apoyar los aprendizajes del alumnado e integrar también a quienes presentan alguna necesidad educativa especial.

En esta misma línea, el 65,6\% señala la existencia de programas de apoyo al estudio para evitar el fracaso escolar, pero en el rango de aplicación permanente solo se da en un 47,3\%. Asimismo, se observa un menor interés (47,3\%) en tener programas para el ocio y el tiempo libre organizados por los establecimientos educacionales y la ejecución de ellos de forma permanente y sistemática solo alcanza un $24,4 \%$. Se revela también en términos satisfactorio $(56,75 \%)$ la existencia de proyectos de innovación educativa para responder a la diversidad y su aplicabilidad en el tiempo de carácter permanente es solo de un $40 \%$.

Un factor relevante en esta dimensión es la presencia del recurso humano especializado en cada uno de los centros educativos, y es así como el 74,3\% de docentes de sala se siente apoyado por los diferentes profesionales especialistas, en la atención al alumnado con necesidades educativas especiales, y el apoyo entregado de carácter permanente, solo alcanza el 59,5\%. 
doi: http://dx.doi.org/10.15359/ree.21-3.17

URL: http://www.una.ac.cr/educare

CORREO: educare@una.cr

Tabla 12: Frecuencia y existencia de recursos del establecimiento educacional

\begin{tabular}{|c|c|c|c|c|c|c|c|c|}
\hline \multirow[t]{2}{*}{ Recursos } & \multicolumn{4}{|c|}{ Docentes de aula } & \multicolumn{4}{|c|}{ Profesionales de apoyo } \\
\hline & Existencia & $\%$ & $\begin{array}{l}\text { Frecuencia } \\
\text { de Uso }\end{array}$ & $\%$ & Existencia & $\%$ & $\begin{array}{l}\text { Frecuencia } \\
\text { de Uso }\end{array}$ & $\%$ \\
\hline $\begin{array}{l}\text { Apoyo del profesorado [especialista] en } \\
\text { el aula para estudiantes con dificultades } \\
\text { en alguna asignatura concreta }\end{array}$ & 216 & 73,9 & 165 & 56,5 & 91 & 98,9 & 79 & 85,8 \\
\hline Aulas de recursos & 171 & 58,5 & 131 & 44,8 & 75 & 81,5 & 56 & 60,8 \\
\hline $\begin{array}{l}\text { [Agrupaciones] en función de los } \\
\text { distintos niveles de rendimiento }\end{array}$ & 187 & 64,0 & 128 & 43,8 & 59 & 64,1 & 45 & 48,9 \\
\hline $\begin{array}{l}\text { Planes de acción tutorial para acoger e } \\
\text { integrar al alumnado con NEE }\end{array}$ & 193 & 66,0 & 141 & 48,2 & 61 & 66,3 & 45 & 48,9 \\
\hline $\begin{array}{l}\text { Apoyo al profesorado para la atención } \\
\text { al alumnado con NEE }\end{array}$ & 217 & 74,3 & 174 & 59,5 & 85 & 92,4 & 64 & 69,6 \\
\hline Servicio de traducción (Lengua de señas) & 48 & 16,4 & 15 & 5,1 & 8 & 8,7 & 2 & 2,2 \\
\hline $\begin{array}{l}\text { Servicio de apoyo educativo } \\
\text { domiciliario para estudiantes que pasan } \\
\text { largos periodos de convalecencia en su } \\
\text { domicilio u hospital }\end{array}$ & 42 & 14,3 & 9 & 3,0 & 10 & 10,9 & 2 & 2,2 \\
\hline $\begin{array}{l}\text { Monitores de apoyo al alumnado } \\
\text { con NEE }\end{array}$ & 183 & 62,6 & 82 & 28,0 & 46 & 50,0 & 30 & 32,6 \\
\hline $\begin{array}{l}\text { Docentes [itinerantes] como apoyo al } \\
\text { alumnado dentro y fuera del centro } \\
\text { escolar }\end{array}$ & 63 & 21,5 & 26 & 8,9 & 19 & 20,6 & 12 & 13,1 \\
\hline $\begin{array}{l}\text { Programa de apoyo al estudio para } \\
\text { evitar el fracaso escolar realizado } \\
\text { por monitores o por profesorado del } \\
\text { colegio o liceo }\end{array}$ & 194 & 66,4 & 140 & 47,9 & 58 & 63,0 & 42 & 45,7 \\
\hline $\begin{array}{l}\text { Programa de ocio y tiempo libre } \\
\text { organizado por el colegio o liceo }\end{array}$ & 141 & 48,2 & 73 & 25,0 & 41 & 44,5 & 21 & 22,8 \\
\hline Apoyo de ONG del entorno & 107 & 36,6 & 47 & 16,0 & 30 & 32,6 & 16 & 17,4 \\
\hline $\begin{array}{l}\text { Aula de apoyo pedagógico para } \\
\text { mejorar las competencias lingüísticas } \\
\text { del alumnado }\end{array}$ & 187 & 64,0 & 137 & 46,9 & 73 & 79,3 & 17 & 18,5 \\
\hline $\begin{array}{l}\text { [Apoyo de] alumnado-ayudantes, } \\
\text { del mismo u otro curso [para con los } \\
\text { compañeros y compañeras con NEE] }\end{array}$ & 162 & 55,4 & 94 & 32,1 & 46 & 50 & 30 & 32,6 \\
\hline
\end{tabular}




\begin{tabular}{|c|c|c|c|c|c|c|c|c|}
\hline \multirow[t]{2}{*}{ Recursos } & \multicolumn{4}{|c|}{ Docentes de aula } & \multicolumn{4}{|c|}{ Profesionales de apoyo } \\
\hline & Existencia & $\%$ & $\begin{array}{l}\text { Frecuencia } \\
\text { de Uso }\end{array}$ & $\%$ & Existencia & $\%$ & $\begin{array}{l}\text { Frecuencia } \\
\text { de Uso }\end{array}$ & $\%$ \\
\hline $\begin{array}{l}\text { Programas de acogida, grupos de } \\
\text { acogida o planes de acogida }\end{array}$ & 96 & 32,8 & 41 & 14,0 & 15 & 16,3 & 12 & 13,0 \\
\hline $\begin{array}{l}\text { Vinculación con centros externos de } \\
\text { recursos }\end{array}$ & 142 & 48,6 & 71 & 24,3 & 40 & 43,5 & 36 & 39,1 \\
\hline $\begin{array}{l}\text { Las ... vacantes de profesionales } \\
\text { que intervienen con alumnado con } \\
\text { discapacidad [son] ocupadas por } \\
\text { personal especializado }\end{array}$ & 212 & 72,6 & 169 & 57,8 & 82 & 89,1 & 74 & 80,4 \\
\hline $\begin{array}{l}\text { Proyectos de innovación ... para } \\
\text { atender la diversidad }\end{array}$ & 165 & 56,5 & 117 & 40,0 & 53 & 57,6 & 38 & 41,4 \\
\hline $\begin{array}{l}\text { [Tiene una] formación que responda } \\
\text { [a la atención educativa de la } \\
\text { diversidad] }\end{array}$ & 142 & 48,6 & 92 & 31,5 & 72 & 78,3 & 48 & 52,2 \\
\hline
\end{tabular}

Nota: Basado en Biencinto, et al. (2009, pp. 24-25, ítem 1, 3, 4, 5, 7, 10, 11,12, 18, 19, 21 y 22).

El 14,5\% de los agentes educativos participantes declaran contar con el servicio de personas traductoras (lengua de señas) en sus aulas, sin embargo, solo se utiliza de manera sistemática el 4,4\%; además, indica que el porcentaje de estudiantes con discapacidad auditiva presente en los establecimientos de educación regular es muy bajo, debido a que esta población dispone de escuelas especiales en trastornos de la comunicación y la atención educativa que brindan algunos colegios privados.

En este mismo sentido, el personal docente de aula señala en un 14,3\% contar con el apoyo educativo domiciliario para aquel alumnado que se encuentran en situaciones de salud que requieren largos períodos de convalecencia, sin embargo, la realización de este apoyo de manera permanente solo alcanza el 3\%, debido a la situación de salud que presentan el estudiantado.

Actualmente, son pocos los establecimientos que cuentan con la presencia de manera itinerante de profesionales con especialidad (21,3\%) en la atención a la diversidad, y su asistencia a los centros educativos de manera frecuente es de un 9,8\%, en opinión de los grupos participantes.

El profesorado de aula y los grupos profesionales de apoyo cuentan con los monitores de apoyo (59,6\%) para el alumnado que presenta NEE, pero su utilización de forma más permanente solo alcanza el 29,1\%; así también ocurre con el alumnado-ayudante del mismo curso o de un curso superior, para apoyar a sus pares que presentan necesidades educativas, que se declara como existencia en los colegios de un $54,1 \%$, pero se contrapone al mismo porcentaje que señala que nunca se utiliza este recurso. 
doi: http://dx.doi.org/10.15359/ree.21-3.17

URL: http://www.una.ac.cr/educare

CORREO: educare@una.cr

Las escuelas y liceos mantienen procesos de vinculación con centros externos y ONG que fluctúan en un $41,5 \%$ y el uso de los aportes y recursos materiales y humanos que estas instituciones realizan solo se utilizan en un $33,8 \%$.

Las plazas y vacantes destinadas a especialistas que apoyan psicopedagógicamente al alumnado que presenta discapacidad son ocupadas en un $72,6 \%$ por profesionales pertinentes tales como docente de educación especial, de fonoaudiología y psicología y con una frecuencia de trabajo específico de intervención educativa que se ubica en el $57,8 \%$, entre el rango de siempre y bastante, en opinión de docentes de aula.

Finalmente, se destaca que los cuerpos docentes de aula perciben que la formación que tienen les permite responder de manera insuficiente a los actuales desafíos educacionales en relación con la atención educativa de la diversidad (48,6\%), a diferencia de los grupos profesionales de apoyo que declaran tener un buen nivel (78,3\%) de preparación en esta área para apoyar a docentes de aula y los procesos relacionados con la atención a la diversidad.

\section{Dimensión nivel de rendimiento}

Esta dimensión solo es considerada por el personal docente de aula, conformando por un total de 292 participantes, pertenecientes a 30 establecimientos educacionales de dependencia municipal y que se ubican en las comunas de Talca y Curicó de la región del Maule.

Tabla 13: Frecuencia de la dimensión nivel de rendimiento

\begin{tabular}{lcc}
\hline Nivel de rendimiento & Docentes de apoyo \\
\cline { 2 - 3 } & $\mathrm{f}$ & $\%$ \\
\hline Los niveles de logro se adaptan según las necesidades del grupo & 240 & 82,9 \\
$\begin{array}{l}\text { El rendimiento o progreso de cada estudiante solo está en función de sus propias } \\
\text { capacidades y del ajuste del programa a estas. }\end{array}$ & 227 & 77,7 \\
$\begin{array}{l}\text { Existen claras diferencias entre las competencias adquiridas, aunque todo el grupo aprenda } \\
\text { Los objetivos básicos a alcanzar son los mismos para todo el grupo. }\end{array}$ & 230 & 78,7 \\
$\begin{array}{l}\text { Existen objetivos básicos y comunes para la totalidad así como objetivos individuales } \\
\text { para cada estudiante }\end{array}$ & 229 & 79,1 \\
\hline
\end{tabular}

Nota: elaboración propia. 
Sus opiniones (Tabla 13) sobre la perspectiva de atención a la diversidad, respecto de los resultados de aprendizaje que obtiene el estudiantado y las consideraciones que realizan en la relación de los objetivos propuestos y las competencias que adquieren en función de las capacidades individuales muestran porcentajes de aprobación por sobre el 75\%.

\section{Diagnóstico}

Esta dimensión es respondida solo por los grupos profesionales de apoyo que se desempeñan en escuelas/liceos en la atención a niños, niñas y jóvenes que presentan necesidades educativas especiales de carácter transitorio y permanente, y que participan del programa de integración escolar (ver Tabla 14).

Las personas participantes declaran la importancia del diagnóstico para identificar las diferencias $(96,7 \%)$ y ajustar la respuesta educativa que asegure su participación en el proceso escolar. Asimismo, destacan como beneficio de este proceso evaluativo la posibilidad de agruparlos para optimizar los apoyos y atención educativa en relación directa con sus necesidades y requerimientos específicos e individuales $(93,5 \%)$.

Tabla 14: Frecuencia de la dimensión diagnóstico

\begin{tabular}{lcc}
\hline \multicolumn{1}{c}{ Diagnóstico } & Profesionales de apoyo \\
\cline { 2 - 3 } & $\mathrm{f}$ & $\%$ \\
\hline $\begin{array}{l}\text { El diagnóstico del alumnado se basa en sus capacidades cognitivas básicas para } \\
\text { hacer grupos diferenciados. }\end{array}$ & 73 & 79,3 \\
$\begin{array}{l}\text { El diagnóstico del alumnado se basa en sus necesidades cognitivas y actitudinales. } \\
\text { Es necesario identificar al alumnado con el fin de asignarles la respuesta educativa } \\
\text { más adecuada. }\end{array}$ & 82 & 89,1 \\
$\begin{array}{l}\text { Es necesario identificar las aptitudes y estilos que permiten alcanzar los objetivos con } \\
\text { el fin de utilizar estrategias docentes adecuadas. }\end{array}$ & 91 & 98,9 \\
$\begin{array}{l}\text { Diagnosticar es fundamental para distinguir entre alumnado dentro de la norma y } \\
\text { alumnado especial. }\end{array}$ & 78 & 84,8 \\
$\begin{array}{l}\text { El diagnóstico es fundamental para agrupar al alumnado de esta forma atender a sus } \\
\text { necesidades educativas. }\end{array}$ & 86 & 93,5 \\
$\begin{array}{l}\text { El diagnóstico es fundamental para identificar las diferencias y poder así ajustar la } \\
\text { respuesta educativa. }\end{array}$ & 89 & 96,7 \\
\hline
\end{tabular}

Nota: Elaboración propia. 
doi: http://dx.doi.org/10.15359/ree.21-3.17

URL: http://www.una.ac.cr/educare

CORREO: educare@una.cr

En este mismo sentido valoran positivamente la aplicación de pruebas e instrumentos de diagnósticos de carácter más integral, para elaborar y plantear un perfil de competencias y capacidades, que permitan orientar, de manera adecuada y pertinente, los objetivos de aprendizaje y la selección de estrategias docentes ajustadas a su realidad escolar.

Sin embargo, el profesorado de curso se plantea desde un enfoque centralmente menos educativo y más clínico, cuando estima (89\%) que estos procesos se realizan desde las necesidades cognitivas y actitudinales que muestra el estudiantado; además, explican $(84,8 \%)$ que es imprescindible diferenciar, al alumnado que se ubica dentro de la norma y el que necesita de recursos y de apoyos específicos.

\section{Conclusiones}

Los resultados de esta investigación dan cuenta de las percepciones de los agentes educativos - personal docente directivo, docentes de aula y profesionales de apoyo-, respecto de cómo perciben la atención a la diversidad y las respuestas que brindan como comunidad educativa a todo el estudiantado que se encuentra en establecimientos educacionales de dependencia municipal.

Estos centros educativos atienden los niveles de enseñanza de pre-básica, básica y media de dos comunas de la región del Maule, y participan del programa de integración escolar que se adscribe a los beneficios de subvención escolar para la educación especial a través del Decreto N.`170 (MINEDUC 2009a) regulado por la Ley Núm. 20.201 (MINEDUC, 2007).

Lasescuelas y liceos participantes muestran un desarrollo positivo y significativo en la variable de contexto en la dimensión educativa de la atención a la diversidad, se observa su tránsito desde enfoques integracionistas hacia unos cada vez más inclusivos. Los distintos agentes educativos ponen de relieve la importancia en el abordaje del quehacer pedagógico de calidad y con equidad, donde todas las personas se sienten participes y comprometidas con las instituciones escolares donde se desempeñan y explicitan que las diferencias son consideradas un valor.

Sin embargo, dejan constancia de las necesidades de formación continua en áreas específicas de la diversidad referidas al conocimiento y comprensión de aspectos teóricos y de la praxis educativa, para responder de mejor modo a los requerimientos de todo el estudiantado, independiente de sus características individuales. Además refieren aspectos de organización escolar en la existencia y uso de recursos materiales y humanos; la desvinculación de instituciones del entorno escolar que pueden aportar en recursos y apoyos específicos a toda la comunidad educativa.

Se debe hacer notar que el sistema escolar chileno está fundado en un paradigma centralizado y homogeneizador en donde las diferencias se visualizan más bien como 
un problema; sin embargo, desde finales del siglo pasado se viene movilizando hacia consideraciones de apertura, de participación, de tolerancia y de mayores democratizaciones en las diversas organizaciones escolares.

\section{Referencias}

Biencinto-López, C., González-Barbera, C., García-García, M., Sánchez-Delgado, P. y MadridVivar, D. (2009). Diseño y propiedades psicométricas del AVACO-EVADIE. Cuestionario para la evaluación de la atención a la diversidad como dimensión educativa en las instituciones escolares. RELIEVE, 15(1), 1-36. Recuperado de http://www.uv.es/RELIEVE/ v15n1/RELIEVEv15n1 4.pdf

Delors, J. (Presidente). (1997). La educación encierra un tesoro. Informe a la UNESCO de la Comisión Internacional sobre la Educación para el siglo XXI. Madrid: Santillana.Recuperado de https:// es.slideshare.net/marrisan/jacques-delors-la-educacin-encierra-un-tesoro-informeunesco

Barreiro, J. (1969). Educación y concienciación en P. Freire (Autor), La educación como práctica de la libertad (pp. 7-19). México: Siglo XXI.

Fundación Nacional de Discapacitados (s. f.). Discapacidad en Chile. Recuperado de http://www. fnd.cl/discapacidadenchile.html

Gobierno de Chile (1998). Constitución política de la República de Chile de 1980. Chile: Editorial Jurídica de Chile.

Ministerio de Educación de Chile (MINEDUC). (2015). Diversificación de la enseñanza. Decreto N. $83 / 2015$. Aprueba criterios y orientaciones de adecuación curricular para estudiantes con necesidades educativas especiales de educación parvularia y educación básica. Recuperado de http://www.educaciónespecial.mineduc.cl/usuarios/edu.especial/File/2015/Decreto\%20 83-2015.pdf

Ministerio de Planificación. (2010). Ley Núm. 20.422. Establece normas sobre igualdad de oportunidades e inclusión social de personas con discapacidad". Recuperado de http:// www.leychile.cl/Navegar?idLey $=20422$

Ministerio de Educación (MINEDUC). (2009a). Decreto Supremo N. ${ }^{\circ} 170$ "Fija normas para determinar los alumnos con necesidades educativas especiales que serán beneficiarios de las subvenciones para educación especial". Recuperado de http://www.mineduc.cl/ usuarios/edu.especial/doc/201304231500550.DEC200900170.pdf 
doi: http://dx.doi.org/10.15359/ree.21-3.17

URL: http://www.una.ac.cr/educare

CORREO: educare@una.cr

Ministerio de Educación (MINEDUC). (2009b). Ley Núm. 20.370. Establece la ley general de educación. Recuperado de http://www.leychile.cl/Navegar?idNorma=1006043

Ministerio de Educación (MINEDUC). (2008). Ley Núm 20.248. Establece ley de subvención escolar preferencial. Recuperado de file:///Users/FAMPENABADCAMACHO/Downloads/ LEY-20248 01-FEB-2008.pdf

Ministerio de Educación (MINEDUC). (2007). Ley Núm. 20.201. Modifica el DFL N. 2 de 1998, de Educación, sobre subvenciones a establecimientos educacionales y otros cuerpos legales. Recuperado de https://www.leychile.cl/Navegar?idNorma=263059

Morin, E. (2000). Los siete saberes necesarios para la educación del futuro. Barcelona: Paidós. Recuperado de http://unesdoc.unesco.org/images/0011/001177/117740so.pdf

Organización Mundial de la Salud (OMS) y Banco Mundial (2011). Informe mundial sobre la discapacidad [Resumen]. Ginebra: Ediciones de la OMS. Recuperado de http://www.who. int/disabilities/world report/2011/summary es.pdf?ua=1

Real Academia Española (RAE). (2014). Diccionario de la lengua española (23a ed.). Recuperado de http://dle.rae.es/?id=EObOPXH 